\section{Dr. Ludvigsson, et al reply}

To the Editor:

We thank Park, et al for their interest ${ }^{1}$ in our report ${ }^{2}$. We agree that the association between SLE and celiac disease (CD) is multifactorial, at both genetic and environmental levels, and merits further study. Interleukin 21 (IL-21), an important cytokine for B cell growth and differentiation, needs to be examined in systemic lupus erythematosus (SLE) and CD, but multiple cytokine pathways are associated with these diseases. The pathogenic role of this cytokine is not exclusive to these 2 diseases, and aberrations in IL-21 are described in rheumatoid arthritis, multiple sclerosis, and Crohn disease. The contribution of IL-21 to the disease process may be complex because of its dual proinflammatory and antiinflammatory effects $^{3}$. IL-21 has a biphasic role in the BXSB-Yaa mouse model of SLE; early treatment with IL-21 receptor-Fc fusion protein led to decreased survival, whereas late treatment was beneficial, but no differences were found in the severity of nephritis between the groups ${ }^{4}$. The authors suspected an early beneficial effect of IL-21 in expanding CD8+ suppressor $\mathrm{T}$ cells, whereas the late benefit was felt to be secondary to its effect on $\mathrm{T}$ follicular helper cells that promote humoral immunity. In CD, IL-21 has a disease-promoting effect, but it is likely in concert with other cytokines such as IL-15 $5^{3}$. In vitro studies show that IL-21 suppresses the maturation of dendritic cells and downregulates the expression and activation of the NKG2D receptor in human natural killer and CD8+ T cells ${ }^{5}$; however, these effects are overcome by IL-15. IL-15 promotes chronic intestinal inflammation through its effect on the transforming growth factor- $\beta$ pathway, and blocking IL-15 may be beneficial ${ }^{6}$.

Although IL-21 is an attractive therapeutic target, more work is needed to understand its role in the pathogenesis of autoimmune diseases.

JONAS F. LUDVIGSSON, MD, PhD, Clinical Epidemiology Unit, Department of Medicine, Karolinska University Hospital and Karolinska Institutet, Stockholm, Department of Pediatrics, Örebro University Hospital, Örebro University, Örebro, Sweden, Division of

Gastroenterology and Hepatology, Department of Medicine, Mayo Clinic

College of Medicine, Rochester, Minnesota, USA; ALBERTO

RUBIO-TAPIA, MD, Division of Gastroenterology and Hepatology, Department of Medicine, Mayo Clinic College of Medicine; VAIDEHI CHOWDHARY, MD, Division of Rheumatology, Department of Medicine, Mayo Clinic College of Medicine; JOSEPH A. MURRAY, MD, Division of Gastroenterology and Hepatology, Department of Medicine, Mayo Clinic College of Medicine; JULIA F. SIMARD, ScD, Clinical

Epidemiology Unit, Department of Medicine, Karolinska University Hospital and Karolinska Institutet. Address correspondence to Dr. J.F. Ludvigsson, Department of Pediatrics, Örebro University Hospital, 701 85 Örebro, Sweden. E-mail: jonasludvigsson@yahoo.com

\section{REFERENCES}

1. Park SW, Park SJ, Shin JI. Another possible underlying mechanism for the positive association between celiac disease and systemic lupus erythematosus: The role of interleukin 21 [letter]. J Rheumatol 2013;40:1619.

2. Ludvigsson JF, Rubio-Tapia A, Chowdhary V, Murray JA, Simard JF. Increased risk of systemic lupus erythematosus in 29,000 patients with biopsy-verified celiac disease. J Rheumatol 2012;39:1964-70.

3. Meresse B, Verdier J, Cerf-Bensussan N. The cytokine interleukin 21: A new player in coeliac disease? Gut 2008;57:879-81.

4. Bubier JA, Bennett SM, Sproule TJ, Lyons BL, Olland S, Young DA, et al. Treatment of BXSB-Yaa mice with IL-21R-Fc fusion protein minimally attenuates systemic lupus erythematosus. Ann NY Acad Sci 2007;1110:590-601.

5. Brandt K, Singh PB, Bulfone-Paus S, Ruckert R. Interleukin-21: A new modulator of immunity, infection, and cancer. Cytokine Growth Factor Rev 2007;18:223-32.

6. Benahmed M, Meresse B, Arnulf B, Barbe U, Mention JJ, Verkarre $\mathrm{V}$, et al. Inhibition of TGF-beta signaling by IL-15: A new role for IL-15 in the loss of immune homeostasis in celiac disease. Gastroenterology 2007;132:994-1008.

J Rheumatol 2013;40:9; doi:10.3899/jrheum.130623 